\title{
Remember-All Based Frontier Allocation for Multi- Robot Coverage in Unknown Environments
}

\author{
Sushil Parti ${ }^{1}$ and Dan $\mathrm{Wu}^{2}$ \\ ${ }^{1}$ Sushil Parti, MSc, School of Computer Science, University of Windsor, Windsor N9B 3P4, Canada \\ ${ }^{2}$ Dan Wu, Asst. Professor, School of Computer Science, University of Windsor, Windsor N9B 3P4, Canada
}

\begin{abstract}
Robots are being increasingly used for coverage tasks which were earlier considered too dangerous or monotonous to be performed by humans such as interplanetary exploration, search \& rescue missions, etc. Out of all the multi-robot coverage approaches, the frontier based approach is one of the most widely used. Most of the coverage approaches developed so far face the issue of frontier duplication and require access to maps of the environments prior to coverage. In this work, a new frontier based strategy for multi-robot coverage in unknown environments is developed. This new strategy tries to remember and manage all the frontiers discovered so far. It is scalable to multiple robots and does not require prior access to the maps. It also uses a new frontier allocation and coordination strategy, which reduces the frontier duplication and improves the efficiency of coverage.
\end{abstract}

Keywords-robot coverage; autonomous robots; frontier coverage; unknown environments; ROS

\section{INTRODUCTION}

The coverage problem is one of the most fundamental problems in robotics, where the main goal is to cover the entire environment. There are many applications such as search and rescue [1], space exploration [2], ocean floor mapping [3] and painting [3] where using robots for coverage could provide better performance at a much lower cost.

Robots can be categorized into two major categories: remote controlled and autonomous robots. Autonomous robots make most of their decisions on their own, while in case of remote controlled robots, a human operator is required. Due to the lack of a human operator, autonomous robots are usually much faster and cheaper than remote controlled robots. In this paper, the term robot is being used to denote autonomous robots.

The usage of multiple robots in a system can bring the additional advantages of increased performance and robustness [4], but it increases the overall complexity and cost of the project. In addition, using multiple robots in a project normally requires:

- A dedicated communication network so that robots can exchange information.

- A coordination strategy that allows the user to take full benefit of multiple robots.

Another major facet of a multi-robot system is the type of environment where it is being used. In this work, our main focus is on unknown environments. Unknown environments are areas about which robot has no information. The usage of unknown environment allows the system to be more flexible and faster than the systems which only work in known environments, as no auxiliary missions are required to create a map. However, in order to perform coverage in unknown environments the following requirements need to be met [5]:

- A Simultaneous Localization and Mapping (SLAM) module is required

- A Map Merging module is required which can create a global map from the local maps of individual robots

- Frontier Detection Strategy

- Frontier Allocation Strategy

Once the basic requirements are met, we need a coverage strategy to guide the robots in such a manner that they cover the whole environment in an optimal manner. In this paper, we consider the problem of multi-robot coverage in unknown environments. In order to improve the efficiency of the overall coverage process, the robots should spread out across the environment and no robot should cover an area which is already covered by another robot.

In this work, we propose a new frontier based coverage strategy - Remember-All Based Frontier Coverage, to perform multi-robot coverage in unknown environments. In the proposed strategy, SLAM is used to simultaneously localize the robots and to create local maps of the environment. Then, a map merging module is used to merge all the local maps into a global map. Once a global map has been created, cellular decomposition is used to divide it, into cells of the same size and shape (occupancy grid). The robots then use the proposed remember-all based frontier coverage strategy to decide in what order they should cover these cells.

The proposed strategy is simulated and tested using ROS and Stage in various custom built environments created to resemble real world scenarios. As it can be observed from the experiments, using the proposed strategy the robots are able to cover a given environment faster and with much less overlap with other robots.

The rest of the paper is organized as follows. Section II introduces the technical concepts in the field of autonomous coverage using multiple robots. Section III summarizes existing frontier based approaches followed by detailed discussion of 
our proposed approach in section IV. Section V presents the experiments followed by conclusions in section VI.

\section{BACKGROUND}

This section discusses some basic concepts and provides a high level overview related to the field of multi-robot coverage in unknown environments.

\section{A. Major Challenges}

In order to solve the problem of multi-robot coverage, we need to answer the following four major questions from the robot's perspective [6]:

\section{1) Where am I?}

Before a robot can start coverage of an environment, the first thing it needs to know is its location. Localization is the process of estimating a robot's current position and orientation in relation to an external frame of reference. In [7], the author states that the problem of localization can be reduced to the problem of coordinate transformation, where the robot's own coordinate system is transformed to a global coordinate system.

2) What does my world look like?

In order for the localization to be successful, the estimated location of the robot should be in respect to some fixed frame of references such as landmarks. Mapping is the process of creating a list of objects in an environment with their respective properties [7].

The above two questions are related to each other and puts one in a state of quandary. Unless the robot has a map of an environment, how can it localize itself? And without localizing how can a robot create a map of its environment. To solve this problem a technique called Simultaneous Localization and Mapping (SLAM) was developed [7]. In this work, we used particle-based SLAM to localize the robot and create a map of its environment at the same time.

\section{3) Where should be my next destination?}

This particular question is not required for the functioning of the overall coverage strategy, but is responsible for making the overall system faster. If the robots cover the area in a particular order, they may be able to perform coverage much faster than if they cover the area without any plan. The proposed strategy provides answer to this question by telling the robot where the best area to move in at each step is.

4) How should I go to my destination?

Once a destination has been selected, a robot requires a path planning algorithm to find a path from its current position to the selected destination. The proposed strategy uses A* path planning algorithm for this purpose.

\section{B. Traditional Coverage Approaches}

\section{1) Random Approach}

In this approach, the robots move around randomly in the environment [8]. This approach is cheaper and easier to implement due to lower computation cost and not requiring costly sensors.

\section{2) Heuristics Approach}

The heuristic approach employs simple thumb rules like follow the wall, repel away from each other's to perform coverage. But the major disadvantage of this approach is that there is no guarantee of complete coverage [8].

\section{3) Potential Fields}

In this approach, the robots repel each other away analogous to the inverse law of electrostatic potential field until they reach a state of equilibrium. It may also happen that the complete coverage is not achieved in all the cases when the robots reach the state of equilibrium [5].

An improvement of this approach is to make the robots get attracted towards unexplored space and repel from the obstacles. But, the major drawback is that the robots may get trapped in a local minima.

\section{Frontier Approach}

The frontier approach was developed in 1997 by Yamauchi [11], and since then it has become one of the most widely used coverage approaches for multi-robot coverage. In the frontier approach, cellular decomposition is used to divide the target area into smaller cells. Each of these cells is then provided with one out of three values - free, unknown and occupied. The values are calculated based on the sensor measurements from the robot sensor. Frontier cells are those cells which lie at the boundary of known and unknown area.

The whole process of frontier based coverage approach can be divided into three major components:

- Frontier Detection

- Frontier Allocation

- Frontier Navigation

In frontier detection, the frontier cells are detected out of all the cells using techniques such as edge detection and region extraction. Once the frontier cells are identified, the frontier allocation strategy decides the frontiers to be allocated to each robot. The last step is frontier navigation where a path is calculated between the robot's current position and the selected frontier. Our main focus in this research is to design a new frontier allocation strategy - Remember-All based Frontier Allocation Strategy which would be discussed in detail later.

\section{EXISTING FRONTIER ALLOCATION STRATEGY}

This section provides a high level overview of the existing frontier allocation strategies with their advantages and disadvantages.

\section{A. Greedy Based Allocation}

In the Greedy Based approach, the robots are allocated to the nearest unallocated frontiers. 


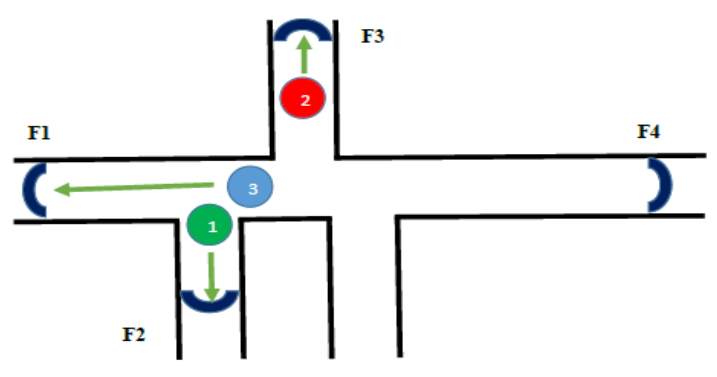

FIGURE I. GREEDY BASED ALLOCATION

Figure I demonstrates the frontier allocation using greedy approach, where all three robots are allocated to separate frontiers. One of the major disadvantage of this approach is that all the robots may get segregated in one area rather than spreading out in the environment.

\section{B. Rank Based Allocation}

The main idea behind rank based allocation is to spread out robots in an environment. The rank based approach uses two key parameters for frontier allocation - Euclidean distance between the robot and frontiers and number of robots near each frontier. First a cost matrix $C$ is created where each element $C_{i,}$ represents the distance between the robot $R_{i}$ and the frontier $F_{j}$. Then, a position matrix $P$ is created where each element $\boldsymbol{P}_{\boldsymbol{y}}$ represents the rank of the robot $R_{i}$ for the frontier $F_{j}$. All the elements of matrix $P$ are calculated using the equation below [9]:

$$
\sum_{\forall_{i \in} \in R, k \neq i, C_{y}<C_{y}}{ }^{1}
$$

Figure II represents the result of frontier allocation using rank based approach. As it can be observed from the figure, all three robots are allocated to separate frontiers and robot 3 is allocated to frontier F4, even though its nearest frontier is frontier F2 as the robots get spread out throughout the environment [9].

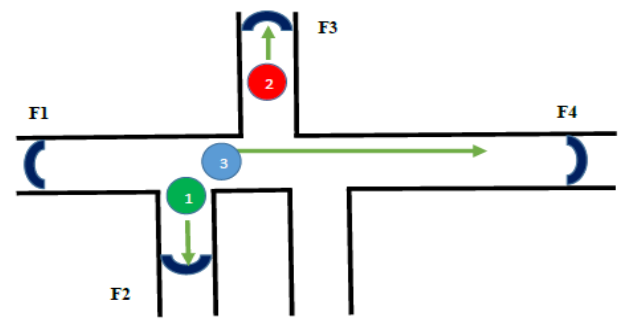

FIGURE II. RANK BASED ALLOCATION

\section{Threshold Based Allocation}

The threshold based approach is an extension of the Rank Based approach where all frontiers in a threshold radius of the robot are marked. All of these marked frontiers are then covered by the robot in the current iteration [5].

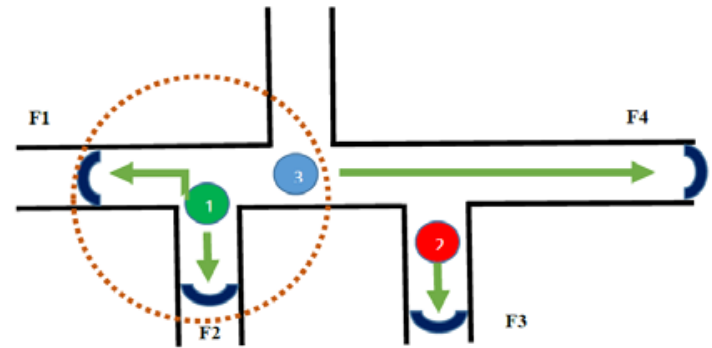

FIGURE III. THRESHOLD BASED ALLOCATION

Figure III represents the frontier allocation using threshold based approach. As it can be observed from the figure, robot 1 gets allocated to all frontiers $F 1$ and $F 2$, as they both lie in its threshold radius. Robot 1 has to cover frontier $F 1$ and $F 2$ before it can participate in the next round of frontier allocation. The other two robots are allocated to other two frontiers.

\section{PROPOSED APPROACH}

As it can be observed from the above coverage strategies, the existing strategies only store the information about the allocated frontiers and discard away the information about the frontiers which are identified in the current iteration but not selected during frontier allocation [5]. The Threshold Based approach tried to solve this problem by defining a threshold, but it coerces the robots to cover all the robots in its threshold radius before the robot can attend to other frontiers, and this may lead to increased overlap.

In [13], the authors used an enhanced frontiers based approach which used storage of frontiers to improve the results. But, it didn't took full advantage of stored frontiers to improve frontier allocations in each iteration. It is our belief that these unallocated frontiers should not be discarded as they may be allocated to the robots in the future. In this section, we introduce a new strategy for frontier allocation, which stores unallocated frontiers and uses them in future iterations.

The proposed Remember-All based frontier allocation strategy aims to store all the information about allocated and unallocated frontiers and used this information in future frontier allocation iterations. This approach will reduce the overall robot overlap and improve performance of the system.

\section{A. Assumptions}

In order to narrow the scope of the implemented system, we have made few assumptions which are consistent with other related and similar research.

1. It is assumed that a communication network is available over the environment.

2. All robots are equipped with fixed static laser sensors with configurable range.

3. All the robot environments are static i.e. there are no changes in the environment when the robots are deliberating.

4. The experiments are conducted for two and three robot systems, but the proposed approach can be easily extended to multi-robot systems with more than three robots. 


\section{B. Coverage Strategy}

In the proposed coverage strategy robots start at random locations in an unknown environment. The proposed strategy can be divided into six major steps to be completed by each robot independently. The robots execute these six steps repeatedly until no new frontiers can be found.

1) Localize the robot and map the environment using SLAM.

2) Create a global map by merging individual maps of the robot.

3) Update the occupancy grid by using individual robot sensor measurements.

4) Identify frontier cells.

5) Allocate one frontier cell to each robot.

6) Navigate the robot to the selected frontier.

Figure IV below depicts the steps of the proposed Remember-All approach for multi-robot coverage for unknown environments. In this work, our main focus is on step 5, where we propose a new frontier allocation strategy with improved communication and coordination strategy.

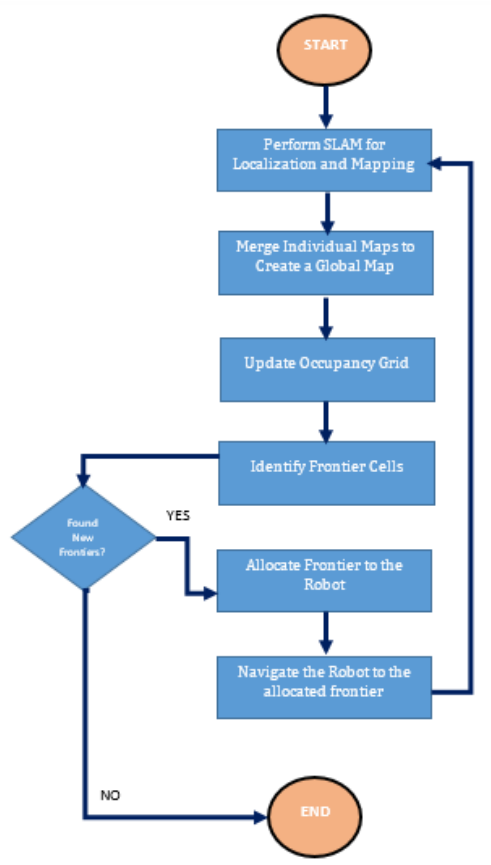

FIGURE IV. STEPS OF THE COVERAGE STRATEGY

\section{Remember-All Based Frontier Allocation}

In the proposed Remember-All approach, each robot maintains three individual lists over the course of the program:

- 'identified_frontiers'

- 'explored_frontiers'

- 'unallocated_frontiers'

The 'identified_frontiers' list is reset at the beginning of each iteration, while the other two lists are persistent till the end of the program. Before the frontier allocation step shown in Figure IV, each robot uses frontier identification technique to identify frontier cells and puts them in its own 'identified_frontiers' list.

The first step in the proposed frontier allocation process is to compare the robot's 'identified_frontiers' list with its own 'explored_frontiers' list, in order to remove any duplicate frontiers. Figure $\mathrm{V}$ depicts the behavior of the proposed strategies in a sample environment, where the robots have identified multiple frontier cells as shown in Figure VI.

At this point, both 'explored_frontiers' and 'unallocated_frontiers' lists are empty for all the robots. The robots then use the rank based approach to allocate one best frontier to each of the robot. The allocated frontiers are represented by shaded cells in Figure VI.

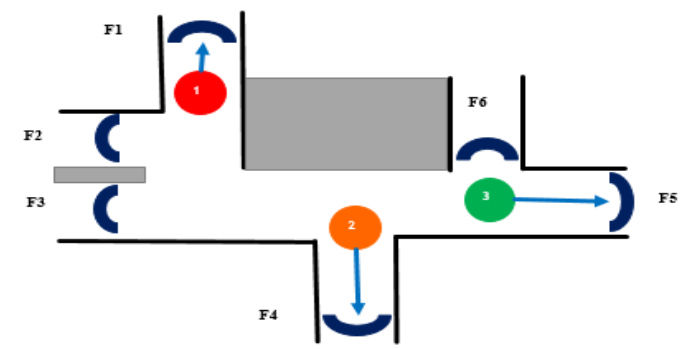

FIGURE V. REMEMBER-ALL BASED APPROACH

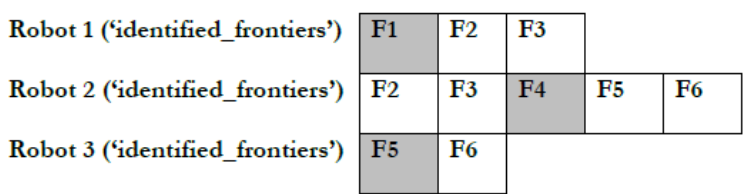

FIGURE VI. IDENTIFIED_FRONTIERS’ LIST

Once the robots are allocated the frontiers, the unallocated frontiers are copied into each robot's 'unallocated_frontiers' list. Figure VII displays this list for this sample environment. The 'unallocated_frontiers' list is used in future frontier allocation iterations, where a configurable amount of frontiers are copied to the 'identified_frontiers' list. This action provides robots with a wider pool of frontiers and allows to mark the frontiers which were identified in earlier iterations, but are not allocated yet.



Each robot then broadcasts the frontier allocated to it, to all other robots, which store the broadcasted frontier in their 'explored_frontiers' list. This storage of explored frontiers helps identify which frontiers are already explored and thus, should not be explored again. Figure VIII depicts the 'explored_frontiers' list for the above example. 


\begin{tabular}{l|l|l|l|}
\cline { 2 - 3 } Robot 1 ('explored_frontiers') & F1 & F4 & F5 \\
\cline { 2 - 4 } Robot 2 ('explored_frontiers') & F1 & F4 & F5 \\
\cline { 2 - 4 } Robot 3 ('explored_frontiers') & F1 & F4 & F5 \\
\cline { 2 - 3 } &
\end{tabular}

\section{FIGURE VIII. EXPLORED FRONTIERS LIST}

Now, in the next iteration, let us suppose that the robot 1 encounters a dead end and is not able to identify any new frontiers. Then, in such a scenario, robot 1 will still be allocated to frontier $F 2$ as it is present in its unallocated frontiers list.

Similarly, robot 2 gets allocated to frontier $F 7$ and robot 3 gets allocated to frontier $F 6$ as displayed in Figure IX. In case a robot is not able to identify any new frontiers and its unallocated frontiers list is also empty, the robot will contact another robot which is nearest to its current position (let's say robot X). Then, a number of recent frontiers (configurable) will be copied from the robot X's 'unallocated_frontiers' list to the robot's ‘identified_frontiers' list.

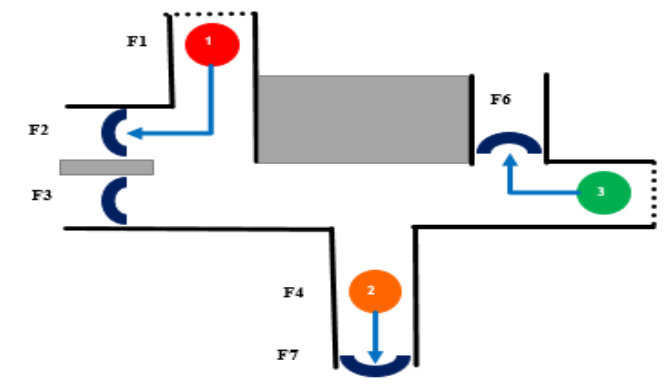

FIGURE IX. REMEMBER-ALL APPROACH (SECOND RUN)

\section{Buddy Approach}

In [12], the authors introduced a problem, where it may happen that when the number of frontiers become less than the number of robots. In that case, some robots may come to a halt as they may believe that coverage is already completed. In this case, we adopt the Buddy approach [12] to address this problem.

In the Buddy approach, the robots with no new frontiers start to follow the leading robot till no new frontiers are found or the leading robot has no more frontiers. If the leading robot finds any new frontiers, they will be share with the follower robots. This approach is useful in environments with long narrow corridors connecting wide open spaces.

\section{EXPERIMENTS \& RESULTS}

This section describes the experiments conducted to test this work and the results obtained. The proposed strategy was implemented using ROS and simulated using Stage 2D. The robots were configured with a hokuyo laser having a 180 degree field of view and a range of 6 meters. The system was tested in three custom built environments of size $34 \times 30$ meters displayed in Figure X.

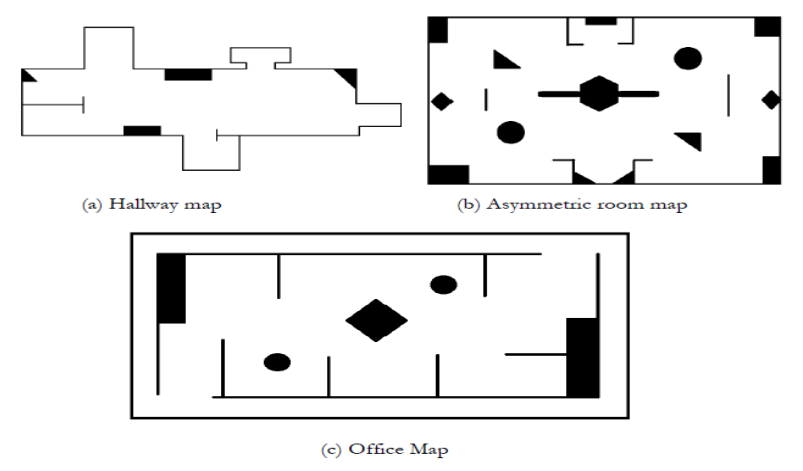

FIGURE X. CUSTOM-BUILT MAPS FOR THE EXPERIMENTS

The proposed strategy was tested with three types of settings:

- Two-Robot System

- Three-Robot System

- Two-Robot System with Distant Starts

For each type of setting, we run our program 10 times for each map and collect the averages of runtime, percentage of duplication frontiers, and frontier coverage percentage for each robot for comparison purpose. Due to the lack of space, we could only report selected results for each setting in one map. However, our complete experiment data [14] shows that the proposed approach runs fastest in all three maps and have no duplicate frontiers.

\section{Two Robot System}

In this set of experiments, two robots start near the center of the map, within close proximity of each other. For each set of experiments, ten runs were performed and results were obtained as average of ten runs. Figure $\mathrm{X}$ displays the average results for the asymmetric room map. As it can be observed from Figure XI, the Remember-All Based approach provides the best result with a runtime of 228.9 seconds and $0 \%$ frontier duplication.

\begin{tabular}{|c|c|c|c|c|}
\hline Frontier Strategy & $\begin{array}{l}\text { Runtime } \\
\text { (in seconds) }\end{array}$ & $\begin{array}{l}\text { Duplicate } \\
\text { Frontiers (\%) }\end{array}$ & $\begin{array}{l}\text { Robot 1 } \\
\text { Frontiers (\%) }\end{array}$ & $\begin{array}{l}\text { Robot 2 } \\
\text { Frontiers (\%) }\end{array}$ \\
\hline $\begin{array}{c}\text { Remember-All } \\
\text { Based }\end{array}$ & 228.9 & 0 & 54.26 & 45.74 \\
\hline Rank Based & 249.1 & 27.99 & 48.17 & 51.83 \\
\hline Threshold Based & 286.9 & 26.83 & 42.05 & 57.95 \\
\hline
\end{tabular}

FIGURE XI. RESULTS FOR ASYMMETRIC MAP

Figure XII depicts the run times for the individual runs for the various coverage strategies in asymmetric room environment. As it can be observed the maximum individual runtime is obtained by Threshold Based approach in run 7, while the minimum runtime is obtained by Rank Based approach in run 2. 


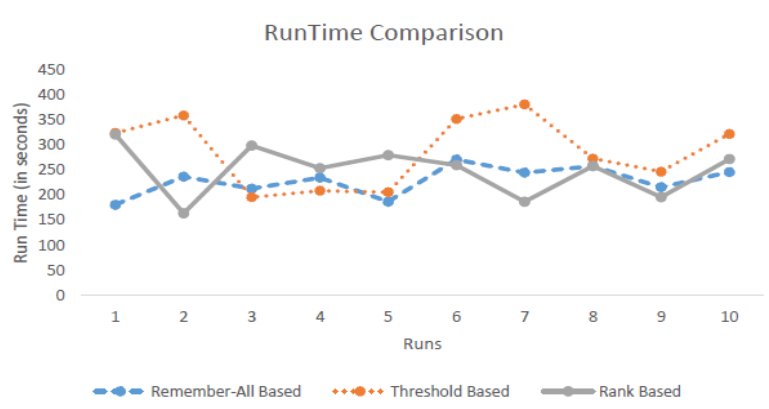

FIGURE XII. RUN TIME COMPARISON FOR ASYMMETRIC MAP

\section{E. Three Robot Systems}

In this set of experiments, three robots start near the center of map, within close proximity of each other and then proceed based on the coverage strategy. Figure XIII displays the average results for the hallway map over the course of ten runs As it can be observed from the Figure XIII, the Remember-All approach performs the fastest with the average runtime of 155 seconds and a frontier duplication of $0 \%$.

In comparison to that, the Threshold Based approach performs worst with an average runtime of 189.9 seconds and a frontier duplication of $34.29 \%$. Fig 14 displays the runtime comparison graph for the three robot system experiment in the hallway map. As it can be observed from the graph in Figure $\mathrm{XV}$ below, the maximum individual runtime is obtained by Threshold Based approach in run 6, while the minimum time was obtained by Remember-All Based approach in run 7.

\begin{tabular}{|c|c|c|c|c|c|}
\hline $\begin{array}{l}\text { Frontier } \\
\text { Strategy }\end{array}$ & $\begin{array}{l}\text { Runtime (in } \\
\text { seconds) }\end{array}$ & $\begin{array}{l}\text { Duplicate } \\
\text { Frontiers (\%) }\end{array}$ & $\begin{array}{l}\text { Robot 1 } \\
\text { Frontiers (\%) }\end{array}$ & $\begin{array}{l}\text { Robot 2 } \\
\text { Frontiers (\%) }\end{array}$ & $\begin{array}{l}\text { Robot 3 } \\
\text { Frontiers (\%) }\end{array}$ \\
\hline $\begin{array}{c}\text { Remember-All } \\
\text { Based }\end{array}$ & 155 & 0 & 59.25 & 23.13 & 17.62 \\
\hline Rank Based & 159.1 & 31.45 & 65.00 & 15.00 & 20.00 \\
\hline Threshold Based & 189.9 & 34.29 & 47.00 & 20.00 & 33.00 \\
\hline
\end{tabular}

FIGURE XIII. RESULTS FOR HALLWAY MAP

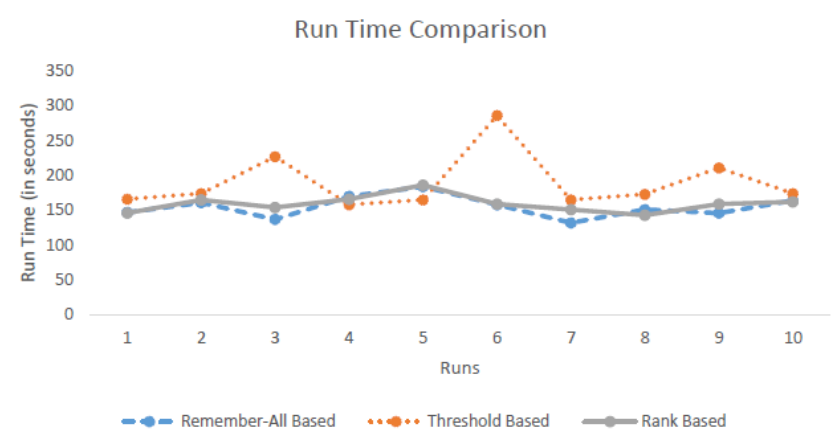

FIGURE XIV. RUNTIME COMPARISON FOR THE HALLWAY

\section{F. Two Robot System with Distant Starts}

In this set of experiments two robots start at distant locations in the map and then proceed further in the environment based on the coverage strategy being used. Figure
$\mathrm{XV}$ displays the results for the two robot system with distant starts for the office environment.

\begin{tabular}{|c|r|r|r|r|}
\hline Frontier Strategy & $\begin{array}{l}\text { Runtime (in } \\
\text { seconds) }\end{array}$ & $\begin{array}{l}\text { Duplicate } \\
\text { Frontiers (\%) }\end{array}$ & $\begin{array}{l}\text { Robot 1 } \\
\text { Frontiers (\%) }\end{array}$ & $\begin{array}{l}\text { Robot 2 } \\
\text { Frontiers (\%) }\end{array}$ \\
\hline $\begin{array}{c}\text { Remember-All } \\
\text { Based }\end{array}$ & 239.30 & 0 & 62.30 & 37.69 \\
\hline Rank Based & 286.30 & 28.17 & 71.43 & 28.57 \\
\hline Threshold Based & 281.90 & 29.35 & 65.87 & 34.13 \\
\hline
\end{tabular}

FIGURE XV. RESULTS FOR OFFICE MAP

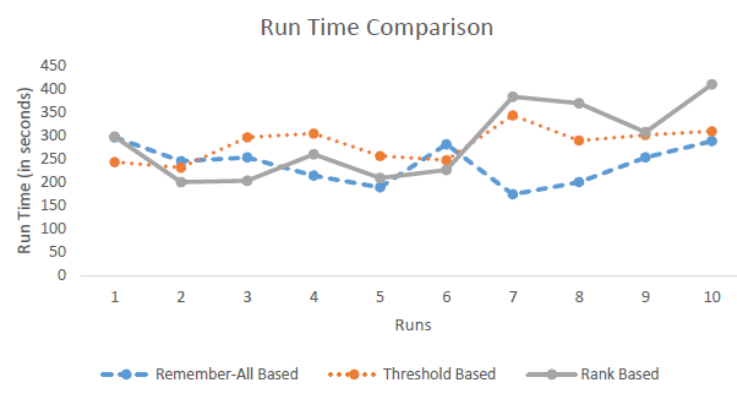

FIGURE XVI. RUN TIME COMPARISON IN OFFICE MAP

As it can be observed from the above figure, the Remember-All Based approach has the lowest average runtime of 239.30 seconds and a frontier duplication of $0 \%$. In comparison the worst performance is by the Threshold Based approach with an average runtime of 281.90 seconds and frontier duplication of $29.35 \%$. Figure XVI displays the individual run time comparison of all the three approaches in the office environment. It can be observed from the above graph, the Remember-All approach has the minimum individual runtime in run 3 , while the maximum runtime is in run 10 by Rank Based approach.

\section{CONCLUSION}

In this work we have developed a new frontier based coverage strategy called Remember-All Based Frontier strategy with a new frontier allocation algorithm. The comparison of the results in section $\mathrm{V}$ demonstrates that the proposed approach performs better than the other two approaches in all three custom-built maps.

The proposed Remember-All approach provides an improvement of $13.3-28.5 \%$ in average runtime in comparison to the Threshold Based approach. In comparison to the Rank Based approach, the proposed approach provides an improvement of $1.8-16.2 \%$. The proposed approach also eliminates frontier duplication in all three environments.

\section{REFERENCES}

[1] D. Hougen et al., "A miniature robotic system for reconnaissance and surveillance,” in Proc. IEEE Int. Conf. Robot. Autom. (ICRA), 2000, pp. 501-507.

[2] D. Apostolopoulos et al., "Robotic antarctic meteorite search: outcomes,” in Proc. IEEE Int. Conf. Robot. Autom. (ICRA), 2001, pp. 4174-4179.

[3] Galceran, Enric, and Marc Carreras. "A survey on coverage path planning for robotics." Robotics and Autonomous Systems 61.12 (2013): 1258-1276. 
[4] Burgard, Wolfram, et al. "Collaborative multi-robot exploration." Robotics and Automation, 2000. Proceedings. ICRA'00. IEEE International Conference on. Vol. 1. IEEE, 2000.

[5] R. S. Dileep Muddu, D. Wu and L. Wu, "A frontier based multi-robot approach for coverage of unknown environments," 2015 IEEE International Conference on Robotics and Biomimetics (ROBIO), Zhuhai, 2015, pp. $72-77$

[6] Leonard, John J., and Hugh F. Durrant-Whyte. "Mobile robot localization by tracking geometric beacons." Robotics and Automation, IEEE Transactions on 7.3 (1991): 376-382.

[7] Thrun, Sebastian, Wolfram Burgard, and Dieter Fox. Probabilistic robotics. MIT press, 2005.

[8] Choset, Howie. "Coverage for robotics-A survey of recent results." Annals of mathematics and artificial intelligence 31.1-4 (2001): 113-126.

[9] Pappas, B.: "Multi-Robot Frontier Based Map Coverage Using the ROS Environment”. PhD thesis, Auburn University (2014)

[10] Sariff, N., and Norlida Buniyamin. "An overview of autonomous mobile robot path planning algorithms." Research and Development, 2006. SCOReD 2006. 4th Student Conference on. IEEE, 2006.

[11] Yamauchi, Brian. "A frontier-based approach for autonomous exploration." Computational Intelligence in Robotics and Automation, 1997. CIRA’97, Proceedings, 1997 IEEE International Symposium on. IEEE, 1997.

[12] Elizondo-Leal, Juan C., Gabriel Ramírez-Torres, and Gregorio Toscano Pulido. "Multi-robot Exploration and Mapping Using Self Biddings and Stop Signals." MICAI 2008: Advances in Artificial Intelligence. Springer Berlin Heidelberg, 2008. 615-625.

[13] Al Khawaldah, Mohammad, and Andreas Nüchter. "Enhanced frontierbased exploration for indoor environment with multiple robots." Advanced Robotics 29.10 (2015): 657-669.

[14] Parti, Sushil. "A New Frontier Based Approach for Multi-Robot Coverage in Unknown Environments." Master's thesis, University of Windsor (2016). 